\title{
Apatinib plus camrelizumab (anti-PD1 therapy, SHR-1210) for advanced osteosarcoma (APFAO) progressing after chemotherapy: a single-arm, open- label, phase 2 trial
}

To cite: Xie L, Xu J, Sun X, et al. Apatinib plus camrelizumab (anti-PD1 therapy, SHR-1210) for advanced osteosarcoma (APFA0) progressing after chemotherapy: a single-arm, open-label, phase 2 trial. Journal for ImmunoTherapy of Cancer 2020;8:e000798. doi:10.1136/jitc-2020-000798

- Additional material is published online only. To view please visit the journal online (http://dx.doi.org/10.1136/jitc2020-000798).

Accepted 14 April 2020

Check for updates

C Author(s) (or their employer(s)) 2020. Re-use permitted under CC BY-NC. No commercial re-use. See rights and permissions. Published by BMJ.

${ }^{1}$ Musculoskeletal Tumor Center, Peking University People's Hospital, Beijing, China ${ }^{2}$ Surgical Oncology, Peking University Shougang Hospital, Beijing, China

${ }^{3}$ Pathology Department, Peking University People's Hospital,

Beijing, China

${ }^{4}$ Radiology Department \& Nuclear Medicine Department, Peking University People's Hospital, Beijing, China

Correspondence to

Dr Wei Guo;

bonetumor@163.com

\section{ABSTRACT}

Background Results of our previous study showed high objective response but short-term activity of apatinib in advanced osteosarcoma. We aimed to investigate the activity of apatinib in combination with camrelizumab in patients with inoperable high-grade osteosarcoma progressing after chemotherapy.

Methods This open-label, phase 2 trial was conducted at Peking University People's Hospital. We enrolled patients with advanced osteosarcoma progressed after chemotherapy. Patients received $500 \mathrm{mg}$ apatinib orally once daily plus $200 \mathrm{mg}$ camrelizumab by intravenous infusion every 2 weeks until disease progression or unacceptable toxicity. The primary endpoint was progression-free survival (PFS) and clinical benefit rate at 6 months, which were based on RECIST V.1.1.

Results 43 patients were enrolled between January 25 and September 4, 2018. With median follow-up time of $48.3(\mathrm{Q} 1, \mathrm{Q} 3,30.6,66.6)$ weeks, $13(30.23 \%, 95 \% \mathrm{Cl}$ $17.2 \%, 40.1 \%$ ) of 43 patients were progression free at 6 months and the 6-month PFS rate was $50.9 \%$ (95\% Cl 34.6\%, 65.0\%). Until final follow-up, the objective response rate was $20.9 \%(9 / 43)$ and two patients with durable disease control were observed. Patients with programmed cell death 1 ligand-1 (PD-L1) tumor proportion score $\geq 5 \%$ and pulmonary metastases tended to have a longer PFS in comparison to the others ( $p=0.004$ and 0.017 , respectively). Toxic effects led to dose reductions, or interruptions, or both in 24 $(55.8 \%)$ of 43 patients and permanent discontinuation in $4(9.3 \%)$ patients. There were no treatment-related deaths.

Conclusions Although the combination of apatinib and camrelizumab seemed to prolong PFS in comparison to single agent apatinib in treating advanced osteosarcoma, it did not reach the prespecified target of 6-month PFS of $60 \%$ or greater. Overexpression of PD-L1 and the presence of pulmonary metastases only were associated with longer PFS.

Trial registration number NCT03359018.

\section{BACKGROUND}

Osteosarcoma, a highly heterogeneous tumor arising from mesenchymal tissues, is highly invasive and prone to hematogenous metastasis in the early stage with a 5-year overall survival (OS) of $71 \%(95 \%$ CI $68 \%$ to $73 \%){ }^{1}$ However, after failure of chemotherapy including high-dose methotrexate (HDMTX), doxorubicin (ADM), cisplatin (DDP), and ifosfamide (IFO), the treatment options are very limited for this orphan disease. ${ }^{2}$ Recently, tyrosine kinase inhibitors (TKIs) targeting angiogenesis have been shown to be effective in inducing objective response and prolonging progression-free survival (PFS) in multiple phase II trials, including sorafenib ${ }^{3}$ and regorafenib. ${ }^{4}$ Our previous phase II trial also revealed that apatinib showed antitumor activity in refractory osteosarcoma by achieving a high response rate of $43.2 \%$ but with a short-lived PFS, ${ }^{5}$ which was consistent with studies involving other TKIs that demonstrate high rates of objective response but with little significant improvement in survival. ${ }^{2-6}$

Osteosarcoma is notable among sarcomas for having a relatively high programmed cell death 1 ligand-1 (PD-L1) expression. ${ }^{7-10}$ Although nivolumab ${ }^{11}$ and pembrolizumab ${ }^{12}$ had ever been used in patients with advanced disease, only a small subset of patients has derived meaningful clinical benefit (online supplementary table S1). Jain ${ }^{13}$ proposed that hypoxia and acidosis during the development of malignant tumors resulted in a decrease in $\mathrm{pH}$, thereby triggering a series of cellular signaling pathways and altering the local tumor microenvironment. Preclinical studies 81415 in our center also showed for osteosarcoma antiangiogenic agents may 
modulate the tumor immunosuppressive microenvironment; thus, combinations of antiangiogenics with immune checkpoint blockers might have synergistic effect. ${ }^{16} 17$

Camrelizumab (SHR-1210, anti-PD-1 antibody) is a high-affinity, humanized, IgG4-א PD-1 monoclonal antibody that was originally researched and developed in China. ${ }^{18}$ We performed a non-comparative, single-arm, open-label, phase II trial to explore the activity and safety of apatinib mesylate in combination with camrelizumab in patients with previously treated advanced osteosarcoma.

\section{METHODS \\ Study design}

This was a prospective, single-arm, open-label, phase II study conducted at a single center to evaluate the safety and efficacy of the combination of apatinib mesylate and camrelizumab in treating patients with inoperable, locally advanced or metastatic osteosarcoma who progressed after chemotherapy.

\section{Study population}

Eligible patients were age 11 years and older with body surface area $>1.2 \mathrm{~m}^{2}$. All patients had histologically confirmed metastatic or locally advanced osteosarcoma, as reviewed by the Pathology Committee of Peking University People's Hospital and were not eligible for curativeintent surgery. Eligible patients had also failed previous systemic chemotherapy, including HD-MTX, ADM, and DDP with/without IFO. Tumors had to be measurable with CT scan or MRI, per RECIST, V.1.1. ${ }^{19}$

Other inclusion criteria were as follows: Eastern Cooperative Oncology Group ${ }^{20}$ performance status of 0 or 1 , life expectancy of 12 weeks or longer, and adequate liver function (defined as total bilirubin $\leq 1 \times$ upper limit of normal (ULN); aspartate aminotransferase and alanine aminotransferase $\leq 2.5 \times \mathrm{ULN}$; international normalized ratio for prothrombin time (PT) $\leq 1.5 \times \mathrm{ULN}$ ), adequate renal function (serum creatinine $\leq 1.5 \times \mathrm{ULN}$ or $\mathrm{Cr}$ clearance $\geq 50 \mathrm{~mL} / \mathrm{min}$ ), and adequate bone marrow function (hemoglobin $\geq 80 \mathrm{~g} / \mathrm{L}$, absolute neutrophil count $\geq 1.5 \times 10^{9}$ cells $/ \mathrm{L}$, platelet count $\geq 75 \times 10^{9}$ cells $/ \mathrm{L}$ ). All patients were assessed by the sarcoma board including a thoracic surgeon with at least 10 years surgical experience. Patients with lung metastases only were carefully assessed for eligibility for metastasectomy, ${ }^{21}$ of whom those who were suitable for surgery were excluded from this study.

Additionally, we excluded patients with severe or uncontrolled medical disorders that could jeopardize the outcomes of the study. These confounding conditions included, cardiac clinical symptoms or disease with left ventricular ejection fraction $<50 \%$, and hypertension that could not be well controlled with antihypertensive drugs. Additional exclusion criteria included the following: weight loss of $20 \%$ or more before illness, brain or leptomeningeal metastasis, surgical procedure or radiotherapy within 4 weeks of enrollment, active gastroduodenal ulcer, previous condition associated with risk of bleeding or requiring anticoagulation, proteinuria or hematuria, denutrition with albuminemia $<25 \mathrm{~g} / \mathrm{L}$, women who were pregnant or breast feeding, other malignancy, positive $\mathrm{HBV} / \mathrm{HCV} / \mathrm{HIV}$ serology, and known allergy to the experimental agents. We also excluded those who had been previously treated with antiangiogenic TKIs and anti-PD-1 or anti-PD-L1 antibodies and those with an active autoimmune disease or syndrome or those who required chronic use of steroids or immunosuppressive drugs.

\section{Treatments}

Patients took $500 \mathrm{mg}$ apatinib orally once daily $30 \mathrm{~min}$ after a meal. Patients were also administered $200 \mathrm{mg}$ camrelizumab intravenously over 30 min once every 2 weeks in a 4 week (28 days) cycle. Neither the research subjects nor the investigators were blinded, and subjects remained on treatment until disease progression defined by iRECIST, ${ }^{22}$ unacceptable toxicity, or withdrawal of consent. Other predefined reasons for patient removal from the trial were as follows: investigator's decision, substantial noncompliance with study requirements, pregnancy, use of illicit drugs or other prohibited substances, development of concurrent illness which could jeopardize clinical status and trial endpoints, or interruption of study drugs for more than 8 weeks.

CT scans of chest, abdomen, and pelvis, as well as bone scan $/{ }^{18}$ Fluorodeoxyglucose Positron Emission Tomography (FDG-PET) scans were performed at baseline and repeated every two cycles. If clinically indicated, tumors at other sites were evaluated by local enhanced CT or MRI. Response was determined by two investigators separately using RECIST V.1.1. ${ }^{19}$ Central radiological review was not routinely done and if investigators had diverging opinions on clinical evaluations, an independent thirdparty radiologists' panel would review the images to verify the results. Responses were confirmed with a second scan at least 4 weeks after the criteria for objective responses were met. Dose modifications were allowed as predefined in the protocol (online supplementary protocol, p 49), and dose delays up to 8 weeks were permitted for adverse events. Whenever feasible, dosing was resumed at the higher dose level. Patients were removed from the study if adverse events did not resolve by palliative treatment and with dose modification/interruption. Patients requiring treatment discontinuation because of adverse events were followed until disease progression or initiation of subsequent therapy and at 8 weeks after the last dose of study drugs. Safety assessments, including laboratory monitoring, were performed during screening and on the first day of every two cycles of therapy. All subjects should have completed the European Organization for Research and Treatment of Cancer Quality of Life Questionnaire (EORTC QLQ-C30) ${ }^{23}$ questionnaires prior to the administration of study drug and received subsequent evaluation of quality of life (QoL) once every two cycles until 
the end-of-treatment visit and then once every 3 months during follow-up.

Biopsies before treatment were optional and obtained before the first drug administration and at the time of disease progression. H\&E slides from all tissue specimens were reviewed by two senior pathologists (SDH and SKK) to identify the presence of malignant cells and to select the best representative tumor blocks from each patient. We performed immunohistochemistry analysis of formalin-fixed paraffin-embedded tissue sections for PD-L1 using an anti-PD-L1 antibody (clone 22C3; Cat\#M3653; DAKO) and Dako Autostainer Link 48 platform. Briefly, all slides were baked at $60^{\circ} \mathrm{C}$, deparaffinized in xylene, and rehydrated with graded ethanols to distilled water. Then, antigen retrieval was performed using DAKO target retrieval solution, high $\mathrm{pH}$, for $4 \mathrm{~min}$ at $99^{\circ} \mathrm{C}$ in a steamer. Non-specific binding was blocked with the Dako EnVision FLEX peroxidase-blocking reagent. Primary antibody was diluted 1:50 using the Dako antibody diluent with background reducing components. All other staining was performed primarily with DAKO series reagents (Cat\#K8002; DAKO). All slides were counterstained with hematoxylin. Specimens were scored by the pathologists using tumor proportion score (TPS), which is the percentage of viable tumor cells showing partial or complete membrane staining at any intensity. The specimen was considered to be PD-L1 positive if TPS $\geq 5 \%$, which was assessed by two pathologists (SDH and SKK).

\section{Study endpoints}

The primary endpoints were PFS and clinical benefit rate (CBR) at 6 months in the intent-to-treat population according to RECIST V.1.1. ${ }^{19}$ The secondary endpoints were OS, objective response rate (ORR), disease control rate (DCR), duration of response (DoR), and toxicity. We also evaluated the QoL of patients and explored the correlation between biomarkers and the efficacy of this therapeutic combination.

PFS was defined as the time from treatment initiation to the time of progression, unacceptable toxicity, or death, whichever came first. CBR was defined as the proportion of patients who did not have disease progression at 24 weeks. OS was defined as the time from treatment initiation to the time of death. ORR was defined as the proportion of patients with complete or partial response (CR or $\mathrm{PR})$. The DCR was the proportion of patients who did not have disease progression $(\mathrm{CR}+\mathrm{PR}+\mathrm{SD})$. DoR was defined as the time from the first documentation of objective response to the time of the first documentation of disease progression or death, whichever came first.

\section{Statistics}

This phase II trial was designed according to Simon's optimum two-stage design with $\alpha=0.05$ and $\beta=0.10$. $\mathrm{P} 0=37 \%$ (null hypothesis) was the PFS rate at 6 months that defined an ineffective drug combination, which was based on the results of our previously published phase II trial, ${ }^{5}$ and $\mathrm{P} 1=60 \%$ or higher (alternative hypothesis) was the PFS rate that defined a promising drug combination. The presence of at least 6 successes in the 12 patients enrolled in the first stage allowed the trial to proceed to the second stage in which 31 more patients were needed to be enrolled for the minimum total of 43 patients $(10 \%$ lost to follow-up calculated).

The intention-to-treat analysis included all patients who received at least one dose of each drug. The evaluable population for treatment activity comprised all patients for whom at least one disease assessment (either clinical or radiological) was performed. We estimated survival endpoints according to the Kaplan-Meier method, with 95\% CIs. RECIST overall responses and disease control were calculated and reported with 95\% CIs. We investigated the effect of PD-L1 expression on survival by comparing survival outcomes with the two-sided Mantle-Cox log-rank test, Fisher's exact test, and the Mantel-Haenszel OR estimate. A log-rank test was used to compare survival curves between different clinical and pathological factors. Statistical analysis was performed using SAS Statistics (V.9.4) and GraphPad Prism (V.5).

\section{Study oversight}

Informed consent was obtained from each patient in Chinese.

\section{RESULTS}

From January 25 to September 4, 2018, a total of 47 patients with chemotherapy-refractory osteosarcoma signed informed consent and were initially screened for this trial (figure 1). Patients who screen failed included one patient who had peritoneum infiltration of osteosarcoma cells and rapidly developed abundant ascites, one patient who withdrew consent before treatment, one who received radiotherapy to his target lesions, and one patient who only had barely intraosseous lesions. Of the 43 enrolled patients, treatment was permanently discontinued in two patients without initial evaluation of efficacy, one of whom developed chronic gastric perforation, and the other had severe pelvic wound dehiscence and needed immediate debridement. They were still included in survival analysis for PFS and OS whereas a total of 41 patients constituted the population for efficacy analysis of log-rank test and detailed information (table 1). All patients were treated according to the protocol. All analyses were performed after the last patient had been followed up for at least 6 months.

After completion of stage $1,7(58.3 \%)$ of 12 patients were progression free at 6 months. Therefore, we enrolled a further 31 patients for stage 2 and 13 (30.2\%) of 43 patients were progression free at 6 months (95\% CI $17.2 \%$ to $40.1 \%$ ). With a median follow-up time of 11.3 months (IQR 7.1-15.5), 41 patients discontinued study drug, of whom 34 had disease progression, 1 radiated remaining lesions, 2 patients withdrew consent for surgeries, 2 dropped out without initial evaluation (mentioned above) and 2 withdrew from the study because of severe 


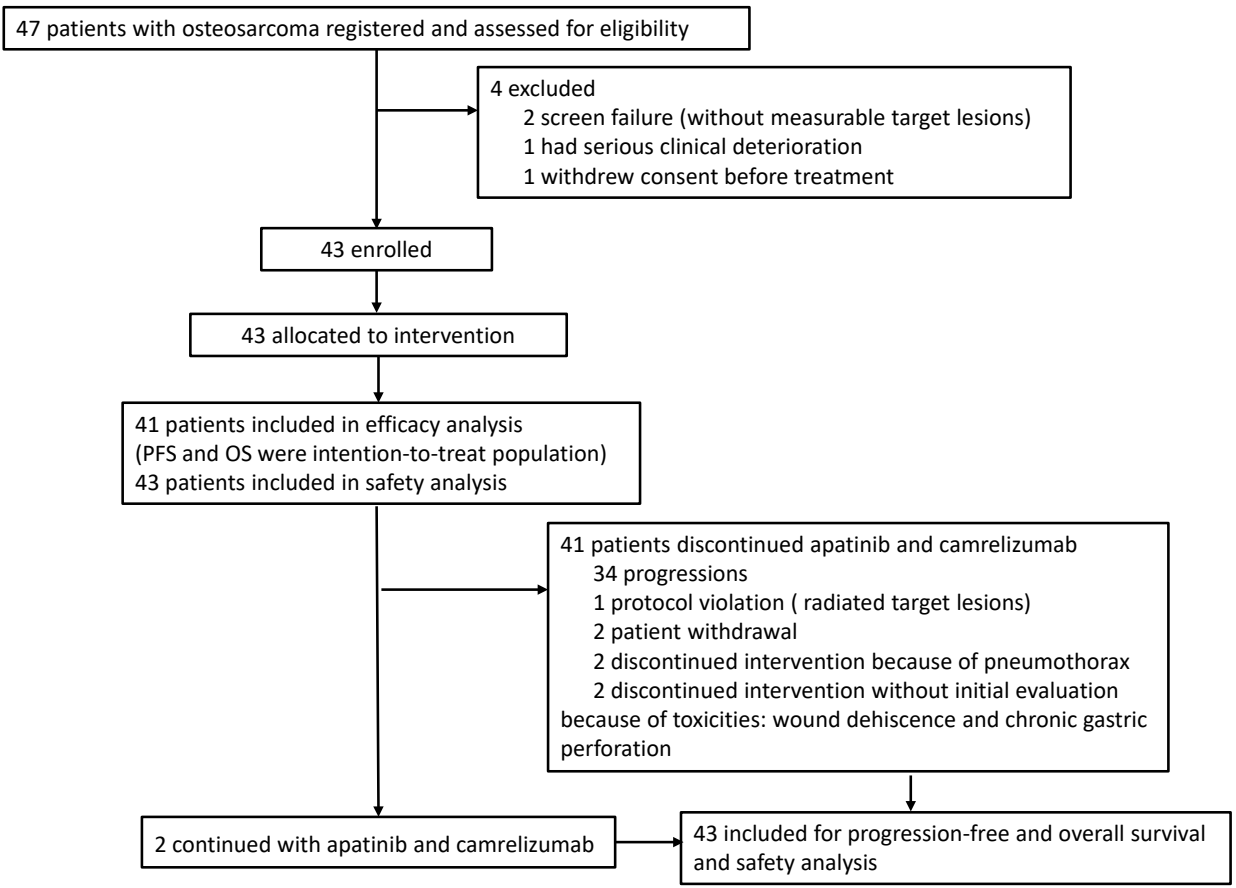

Figure 1 Trial profile. OS, verall survival; PFS, progression-free survival.

pneumothorax combined with infection. The median PFS was 6.2 months (95\% CI 4.0 to 6.9), with 4-month and 6-month PFS rates of $66.3 \%$ (95\% CI $49.8 \%$ to $78.5 \%$ ) and $50.9 \%$ (95\% CI $34.6 \%$ to $65.0 \%$ ), respectively (figure 2 ). With the data cut-off date of October 22, 2019, 12/43 patients $(27.9 \%)$ were alive with disease, $28 / 43(65.1 \%)$ patients had died from disease, and $3 / 43(7.0 \%)$ were lost to follow-up (online supplementary figure $\mathrm{S} 1$ and table S2). A median OS of 11.3 months (95\% CI 8.1 to 14.8 ) was estimated using the Kaplan-Meier method (figure 3). Of all 43 patients, we recorded an overall response rate of $20.1 \%$ (9/43; figure 4; online supplementary figure S2). The median time to response was 1.8 months (95\% CI 1.2 to 2.0) with median DoR was 6.2 months $(95 \%$ CI 3.6 to 8.9). Based on iRECIST, the median PFS was 7.5 months (95\% CI 6.0 to 9.7), and a 6 -month PFS rate of $65.3 \%$ (95\% CI $47.8 \%$ to $78.2 \%$; online supplementary figure S3).

The baseline clinical characteristics and key on treatment laboratory changes were summarized in table 1 . All patients had already received MAP/I chemotherapy with a median number of previous systemic regimens of 1.2 (range 1-3, we defined IFO as the first-line regimen). The male-to-female ratio in the study population was almost 3:1. Baseline $\mathrm{C}$ reactive protein $(\mathrm{CRP})^{24}$ and neutrophil lymphocyte ratio (NLR) ${ }^{25}$ have been reported to be associated with the efficacy of immunotherapy. However, in our study, all patients had NLR $\geq 3 \%$ and $39.0 \%$ (16/41) had higher than normal levels of CRP, which had no obvious impact on PFS. Nevertheless, a significant number of patients $(78.0 \%-85.4 \%$; 32-35/41) developed hypercoagulability during treatment, with decreased PT and activate partial thromboplastin time (APTT) and thrombocythemia with univariate Cox analysis significantly shorting PFS with HRs of 3.61, 5.85, and 4.88, respectively ( $\mathrm{p} \leq 0.001$ for all three) whereas multivariate Cox analysis of these clinical factors demonstrated that only the locations of lesions significantly influenced PFS ( $p$ value of 0.004 ).

Immunohistochemical evaluation of PD-L1 expression was feasible in pretreatment tumor samples from $28(68.3 \%)$ of 41 patients. Of these 28 patient samples, 6 (21.4\%) were PD-L1 positive. Significant statistical difference was found in the PFS of patients with PD-L1 expression less than $5 \%$ and $\geq 5 \%$, with a p value of 0.004 while ORR was not so obvious $(\mathrm{p}=0.11$; Figure 4$)$. We classified participants into three groups according to their lesions at enrollment and found patients with musculoskeletal lesions only had markedly poorer prognosis than those with pulmonary lesions only or combinations of both, with a median PFS of 2.1 (95\% CI 1.8 to 5.7$), 6.9$ (95\% CI 4.8 to 8.1 ), and 5.7 (95\% CI 3.5 to 7.5 ) months, respectively (online supplementary file 2 ).

Until last follow-up, two patients remained on treatment. All 43 patients received a total of 270 cycles of treatment with a median treatment time of 6.2 months (IQR 4.3-9.9) and a median of 6.1 treatment cycles (IQR 4.0-8.0). The frequency of administered full-planned dose of apatinib was $51.2 \%$, and nearly $95 \%$ for camrelizumab (table 2). The median duration for dose interruption throughout the trial was 15.0 days (IQR 3.0-31.5) for apatinib and 11.0 days (IQR 7.0-17.1) for camrelizumab (table 3). Of 43, $28(65 \cdot 1 \%)$ patients have died as of the most recent time of follow-up. Disease progression was the cause of most of these deaths. All treatment-emergent related and unrelated AEs are presented in table 3 (full description in online supplementary table S3). 
Table 1 Demographics

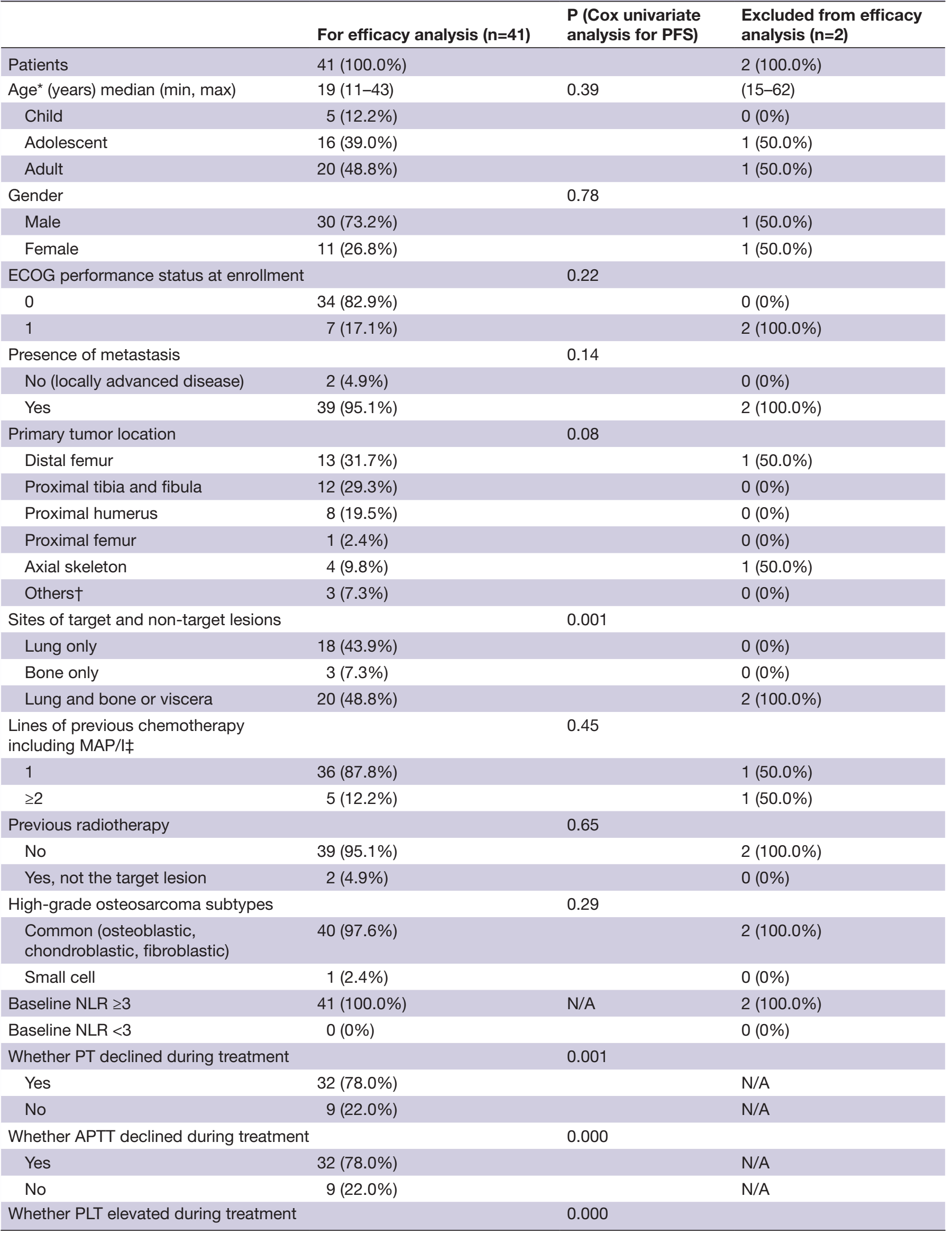


Table 1 Continued

For efficacy analysis $(n=41)$

\begin{tabular}{|c|c|c|c|}
\hline Yes & $35(85.4 \%)$ & & N/A \\
\hline $\begin{array}{l}\text { Whether D-dimer elevated during } \\
\text { treatment }\end{array}$ & & 0.74 & \\
\hline Yes & $22(53.7 \%)$ & & N/A \\
\hline No & $18(43.9 \%)$ & & N/A \\
\hline
\end{tabular}

*Groups defined according to Collins et al: child (0-12 for males and 0-11 for females), adolescent (13-17 for males and 12-16 for females), and adult ( $\geq 18$ for males and $\geq 17$ for females). ${ }^{29}$

†Others including one polycentric osteosarcoma, one located at foot and one located distal radius.

$\ddagger \mathrm{MAP} / \mathrm{l}$, including high-dose methotrexate, doxorubicin, cisplatin with or without ifosfamide. We defined these four agents as first-line chemotherapy.

§Alkaline phosphatase cut-off value according to Bacci et al, defined as: cut-off: 2-10 years 350 IU/L; 10-13 years female 400IU/L; 13-15 years male $500 \mathrm{IU} / \mathrm{L}$; $20-50$ years $100 \mathrm{IU} / \mathrm{L}$; other childhood age $300 \mathrm{IU} / \mathrm{L}$.

AE, adverse events; APTT, activate partial thromboplastin time; ECOG, Eastern Cooperative Oncology Group; N/A, not available; NLR, neutrophil lymphocyte ratio; PFS, progression-free survival; PLT, platelet; PT, prothrombin time; TSH, thyroid-stimulating hormone.

We tracked and recorded self-reported questionnaires about QoL before, during, and after treatment by EORTC QLQ-C30 (version 3.0) ${ }^{26}$ The mean global health status was 67 (IQR 42-83), 67 (IQR 33-83), and 58 (IQR 33-67) at baseline, mean scores on-treatment assessment, and the time of disease progression, respectively. We recorded a trend of improvement in physical and role functioning after treatment, and higher symptom scores in diarrhea and loss of appetite, however without statistical significance $(\mathrm{p}>0.100)$. Off-treatment and on-treatment visit forms were not available in nine and two patients, respectively.

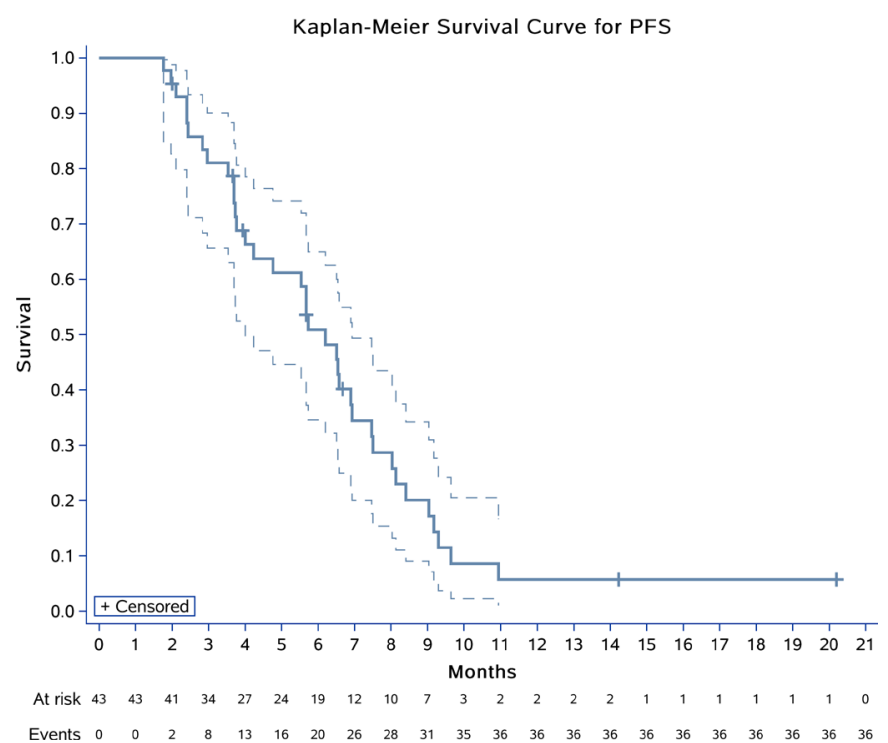

Figure 2 Kaplan-Meier plots for progression-free survival (PFS) in 43 patients (intention-to-treat population).

\section{DISCUSSION}

The findings from this single-arm, open-label, phase II trial revealed that the combination of apatinib and camrelizumab had a median PFS of 6.2 months $(95 \%$ CI 4.0 to 6.9 ) and CBR of $30.2 \%$ (95\% CI $17.2 \%$ to $40.1 \%$ ) for advanced osteosarcoma. However, based on our previous data of single apatinib in osteosarcoma, ${ }^{5}$ the advantage of the combination was not statistically significant, which is less than the prespecified threshold of activity necessary to deem the combination of apatinib and camrelizumab worthy of a phase 3 trial. The lowered ORR may have been as a result of several factors. First, the dose of single apatinib study was much higher than that used in the combination therapy. Most patients $(83.8 \% ; 31 / 37)$ in the former trial were initially treated with apatinib $750 \mathrm{mg}$

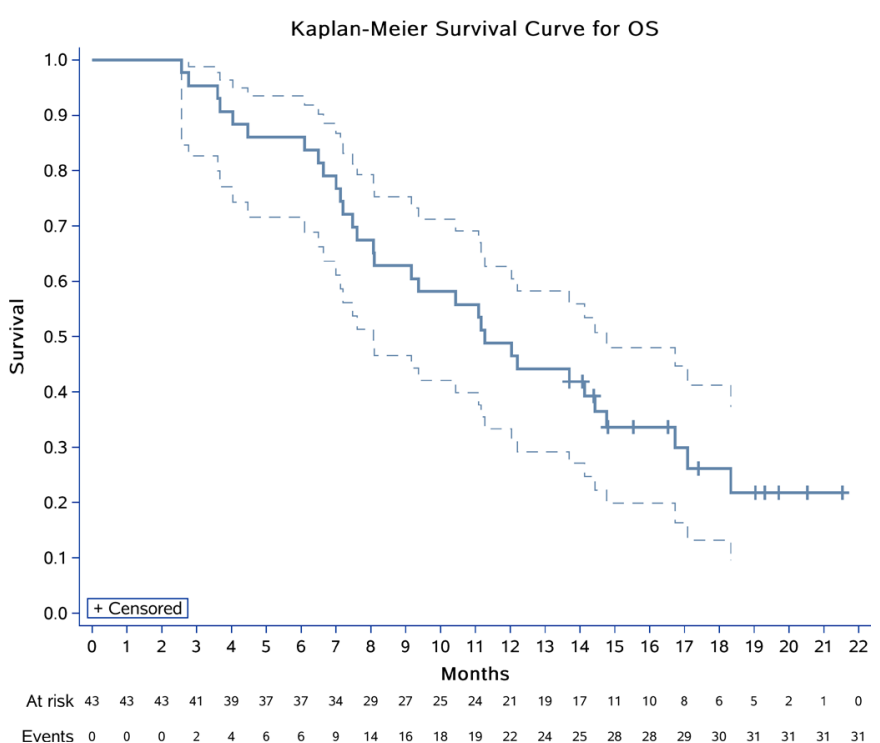

Figure 3 Kaplan-Meier plots for overall survival (OS) in 43 patients (intention-to-treat population). 


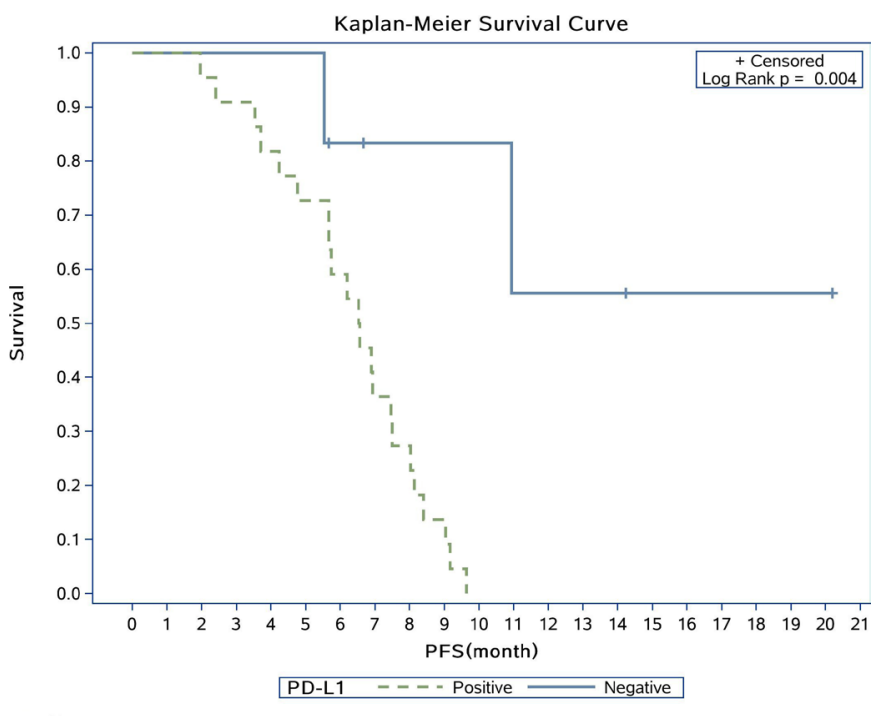

Positive
Negative

Figure 4 Kaplan-Meier plot for progression-free survival (PFS) based on programmed cell death 1 ligand-1 (PD-L1) immunohistochemical expression. Anti-PD-L1 antibodies: clone 22C3; Cat\#M3653; DAKO. Log-rank test $p=0.004$. Crosses indicate censoring.

daily, which was in contrast to the $500 \mathrm{mg}$ in this regimen due to the threat of overlapping toxicities. Second, the portion of pulmonary lesions only was much higher in former study $(73.0 \% ; 27 / 37)$ than in this one $(41.9 \%$; $18 / 41)$, which was relevant as we found that pulmonary lesions were more responsive to treatment than bone lesions. Third, the initial evaluation time was much shorter in the former study (4 weeks) than present study ( 8 weeks), which may have allowed for tumor responses to be missed due to the rapid development of acquired resistance. Finally, camrelizumab might compromise the ORR of apatinib because tumor pseudoprogression in the early stage could also occurred.

Immunotherapy for osteosarcoma has not been reported as effective as other solid tumors, like melanoma, after failures of interferon alfa-2b, ${ }^{27}$ GD2-antibody ${ }^{28}$ and so on. Recently, checkpoint inhibitors have drawn public attention for their relatively high response rates and durable responses. However, from the prospective trials of Alliance A091401 ${ }^{11}$ and SARC028, ${ }^{12}$ we have only observed the inclusion of a few cases of osteosarcoma with even fewer long-term survivors. We are the first to report a prospective trial using combination of TKIs and PD-1 antibody in osteosarcoma. However, in this study, synergistic effect has not been identified in most population and until last follow-up, only two relatively long-term survivors (more than 12 months) have been observed.

Our research efforts are also now geared toward identifying the patients with osteosarcoma who stand to benefit from immunotherapy. With an initial PD-L1 positive expression rate as low as $21.4 \%$ (6/28), we did not observe any ORR benefit but prolonged PFS in patients with PD-L1-expressing tumors. However, this observation indicated that ORR might not be the most appropriate
Table 2 Dose reductions

\begin{tabular}{|c|c|}
\hline & $\begin{array}{l}\text { Number }(\%) \text { or } \\
\text { time (day) }\end{array}$ \\
\hline $\begin{array}{l}\text { Treatment permanently interrupted } \\
\text { because of toxic effects }\end{array}$ & $4 / 43(9.3 \%)$ \\
\hline $\begin{array}{l}\text { Treatment dose reduced/temporarily } \\
\text { interrupted }\end{array}$ & $24 / 43(55.8 \%)$ \\
\hline Treatment temporarily interrupted & $6 / 43(14.0 \%)$ \\
\hline Treatment dose reduced & $21 / 43(48.8 \%)$ \\
\hline \multicolumn{2}{|l|}{ Dose of apatinib +camrelizumab } \\
\hline 500 mg daily +200 mg Q2W initially & $43 / 43(100.0 \%)$ \\
\hline $\begin{array}{l}250 \mathrm{mg} \text { daily }+200 \mathrm{mg} \text { Q2W (-1 dose } \\
\text { level) }\end{array}$ & $21 / 43(48.8 \%)$ \\
\hline $\begin{array}{l}125 \text { mg daily +200 mg Q2W (-2 dose } \\
\text { level) }\end{array}$ & $1 / 43(2.3 \%)$ \\
\hline \multicolumn{2}{|l|}{ Days apatinib held for one interruption } \\
\hline Mean (SD) & $9.0(10.2)$ \\
\hline Median (IQR) & $3.0(2.0-15.0)$ \\
\hline \multicolumn{2}{|l|}{ Days apatinib held during trial } \\
\hline Mean (SD) & $18.0(14.2)$ \\
\hline Median (IQR) & $15.0(3.0-31.5)$ \\
\hline \multicolumn{2}{|l|}{$\begin{array}{l}\text { Days camrelizumab held for one } \\
\text { interruption }\end{array}$} \\
\hline Mean (SD) & $12.7(7.3)$ \\
\hline Median (IQR) & $11.0(7.0-17.1)$ \\
\hline \multicolumn{2}{|l|}{ Days camrelizumab held during trial ${ }^{\star}$} \\
\hline Mean (SD) & $12.7(7.3)$ \\
\hline Median (IQR) & $11.0(7.0-17.1)$ \\
\hline
\end{tabular}

Data are $\mathrm{n}(\%)$, mean (SD), or median (IQR). Camrelizumab was temporary interrupted mainly because of immune-related toxicities judged by investigators. Temporary interruptions of both drugs were usually for several causes: wound dehiscence, diarrhea, blood bilirubin increased and aminotransferase increased and pneumothorax. Of the 43 patients, 21 patients who had reduced apatinib doses to $250 \mathrm{mg}$ were also treated at a dose level of 200 mg Q2W of camrelizumab (-1 dose level). Thrombocytopenia, hand and foot syndrome, oral mucositis or other skin toxic effects, hypertension, and diarrhea were the most common causes for apatinib dose reductions.

*Throughout the trial, camrelizumab had only been held for temporary interruptions for once in all four patients.

maker to hint the efficacy of PD-1 antibody for osteosarcoma because the osteogenic lesions with calcification might not shrink as other solid tumors' soft mass. During the study, we made note of an interesting observation regarding the site of tumor lesions and disease progression, of which we found that patients with pulmonary lesions only had a tendency for longer PFS than those with bone lesions $(p=0.017)$. In the meantime, the occurrence of hypercoagulation in our patients with decreased PT or APTT $(78 \% ; 32 / 41)$ and thrombocythemia $(85.4 \%$; $35 / 41$ ) was also associated with poorer prognosis in terms of PFS. Further studies are also needed to determine if 
Table 3 Adverse events that arose in at least three patients

\begin{tabular}{|c|c|c|}
\hline Adverse event & All, n (\%) & Grade 3/4, n (\%) \\
\hline Sum of all & $43(100.0)$ & $30(69.8)$ \\
\hline Wound dehiscence & $11(25.6)$ & $6(14.0)$ \\
\hline ALP increased & $31(72.1)$ & $4(9.3)$ \\
\hline Blood bilirubin increased & $22(51.2)$ & $4(9.3)$ \\
\hline Hypertriglyceridemia & $32(74.4)$ & $3(7.0)$ \\
\hline Anorexia & $20(46.5)$ & $3(7.0)$ \\
\hline Weight loss & $15(34.9)$ & $3(7.0)$ \\
\hline Pneumothorax & 9 (20.9) & $3(7.0)$ \\
\hline Platelet count decreased & $30(69.8)$ & $2(4.7)$ \\
\hline Diarrhea & $21(48.8)$ & $2(4.7)$ \\
\hline Hand-foot syndrome & $21(48.8)$ & $2(4.7)$ \\
\hline Pain in extremity & $20(46.5)$ & $2(4.7)$ \\
\hline AST increased & $18(41.9)$ & $2(4.7)$ \\
\hline ALT increased & $17(39.5)$ & $2(4.7)$ \\
\hline Leukopenia & $16(37.2)$ & $2(4.7)$ \\
\hline Rash & $14(32.6)$ & $2(4.7)$ \\
\hline Mucositis oral & $11(25.6)$ & $2(4.7)$ \\
\hline Hypertension & $10(23.3)$ & $2(4.7)$ \\
\hline Abdominal pain & 8 (18.6) & $2(4.7)$ \\
\hline Toothache & $6(14.0)$ & $2(4.7)$ \\
\hline Non-cardiac chest pain & $2(4.7)$ & $2(4.7)$ \\
\hline Hypothyroidism & 35 (81.4) & $1(2.3)$ \\
\hline Blood LDH increased & $27(62.8)$ & $1(2.3)$ \\
\hline Proteinuria & $11(25.6)$ & $1(2.3)$ \\
\hline Cough & $10(23.3)$ & $1(2.3)$ \\
\hline Nausea & $8(18.6)$ & $1(2.3)$ \\
\hline Vomiting & $6(14.0)$ & $1(2.3)$ \\
\hline Hemorrhoidal hemorrhage & $6(14.0)$ & $1(2.3)$ \\
\hline Hair color changes & $5(11.6)$ & $0(0.0)$ \\
\hline Fatigue & $4(9.3)$ & $1(2.3)$ \\
\hline $\begin{array}{l}\text { Peripheral } \\
\text { neuroinflammation }\end{array}$ & $3(7.0)$ & $1(2.3)$ \\
\hline
\end{tabular}

ALP, alkaline phosphatase; ALT, alanine aminotransferase; AST, aspartate aminotransferase; LDH, lactate dehydrogenase.

hypercoagulation is an indicator of acquired resistance or just a consequence of high tumor burden.

The occurrence of AEs in this study is consistent with the safety profile of TKIs investigated in patients with advanced osteosarcoma. ${ }^{3-6}$ However, the increased incidence of AEs (of any grade), and in particular for hypothyroidism, thrombocytopenia, anorexia, and diarrhea, may be attributable to camrelizumab.

We are aware of many limitations in this study. We did not include many biomarker-related studies in this trial. For example, the tumor mutation burden was unknown for these patients and the biopsies were optional, which lead to no comprehensive understanding of patients'
PD-L1 status. Additionally, this study was one cohort study of the combination drugs instead of a randomized controlled study for a head-to-head comparison with single apatinib for osteosarcoma, which made this comparison not objective with different patients' baseline states and other factors influencing prognosis.

\section{CONCLUSION}

Although the combination of apatinib and camrelizumab seemed to prolong PFS in comparison to single agent apatinib in treating advanced osteosarcoma, it did not reach the prespecified target of 6-month PFS of $60 \%$ or greater. Until last follow-up, two durable therapeutic effects were observed. Overexpression of PD-L1 and the presence of pulmonary metastases only were associated with longer PFS. More studies are needed to determine biomarkers that may aide in patient selection for this combination.

Acknowledgements The authors thank all the patients and their families for participating in this clinical trial. They also thank Medlink (the third party for data analysis) for interpretation of all the clinical data and statistical analysis of this trial.

Contributors $L X, J X$, and WG designed the study. WG, XT, TY, RY, and JG were responsible for material provision and patient inclusion. LX, JX, XS, and YH collected and assembled data. Pathological reviewing the slides of this trial: KS and DS reviewed the slides of this trial. YL, $L X$, and JX did the clinical evaluation of the trial. $\mathrm{KL}, \mathrm{BZ}$, and TR did the laboratorial work and molecular biological analysis of this study. $L X, J X$, and WG analyzed and interpreted the data. JX and $L X$ analyzed the quality of life. $L X$ wrote the manuscript. All authors gave their final approval of the manuscript. All authors were accountable for all aspects of the work.

Funding This work was supported by Jiangsu HengRui Medicine Co. and the Chinese National Natural Science Foundation (81572633 to WG).

Competing interests None declared.

Patient consent for publication Parental/guardian consent obtained.

Ethics approval This clinical trial was conducted after approval by the local institutional review board and independent ethics committee and done in accordance with the ethical principles derived from the Declaration of Helsinki, International Conference on Harmonization Good Clinical Practice Guidelines and locally applicable laws and regulations on January 24,2018 . Informed consent was obtained from each patient in Chinese.

Provenance and peer review Not commissioned; externally peer reviewed.

Data availability statement Data are available upon reasonable request. All the data belongs to Peking University People's Hospital. URL: https://www.pkuph.cn/. All data generated or analyzed during this study are included in this article and its supplementary files. Under reasonable request, detailed information could be obtained by signing informed consent by e-mail: xie.lu@hotmail.com.

Open access This is an open access article distributed in accordance with the Creative Commons Attribution Non Commercial (CC BY-NC 4.0) license, which permits others to distribute, remix, adapt, build upon this work non-commercially, and license their derivative works on different terms, provided the original work is properly cited, appropriate credit is given, any changes made indicated, and the use is non-commercial. See http://creativecommons.org/licenses/by-nc/4.0/

\section{ORCID iD}

Wei Guo http://orcid.org/0000-0002-4772-6592

\section{REFERENCES}

1 Smeland S, Bielack SS, Whelan J, et al. Survival and prognosis with osteosarcoma: outcomes in more than 2000 patients in the EURAMOS-1 (European and American osteosarcoma study) cohort. Eur J Cancer 2019;109:36-50. 
2 Luetke A, Meyers PA, Lewis I, et al. Osteosarcoma treatment where do we stand? A state of the art review. Cancer Treat Rev 2014;40:523-32.

3 Grignani G, Palmerini E, Dileo P, et al. A phase II trial of sorafenib in relapsed and unresectable high-grade osteosarcoma after failure of standard multimodal therapy: an Italian sarcoma group study. Ann Oncol 2012;23:508-16.

4 Duffaud F, Mir O, Boudou-Rouquette P, et al. Efficacy and safety of regorafenib in adult patients with metastatic osteosarcoma: a noncomparative, randomised, double-blind, placebo-controlled, phase 2 study. Lancet Oncol 2019;20:120-33.

5 Xie L, Xu J, Sun X, et al. Apatinib for advanced osteosarcoma after failure of standard multimodal therapy: an open label phase II clinical trial. Oncologist 2019;24:e542-50.

6 Grignani G, Palmerini E, Ferraresi V, et al. Sorafenib and everolimus for patients with unresectable high-grade osteosarcoma progressing after standard treatment: a non-randomised phase 2 clinical trial. Lancet Oncol 2015;16:98-107.

7 Huang X, Zhang W, Zhang Z, et al. Prognostic value of programmed cell death 1 ligand-1 (PD-L1) or PD-1 expression in patients with osteosarcoma: a meta-analysis. J Cancer 2018;9:2525-31.

8 Zheng B, Ren T, Huang Y, et al. Apatinib inhibits migration and invasion as well as PD-L1 expression in osteosarcoma by targeting STAT3. Biochem Biophys Res Commun 2018;495:1695-701.

9 Sundara YT, Kostine M, Cleven AHG, et al. Increased PD-L1 and T-cell infiltration in the presence of HLA class I expression in metastatic high-grade osteosarcoma: a rationale for T-cell-based immunotherapy. Cancer Immunol Immunother 2017;66:119-28.

10 Koirala P, Roth ME, Gill J, et al. Immune infiltration and PD-L1 expression in the tumor microenvironment are prognostic in osteosarcoma. Sci Rep 2016;6:30093.

11 D'Angelo SP, Mahoney MR, Van Tine BA, et al. Nivolumab with or without ipilimumab treatment for metastatic sarcoma (alliance A091401): two open-label, non-comparative, randomised, phase 2 trials. Lancet Oncol 2018;19:416-26.

12 Tawbi HA, Burgess M, Bolejack V, et al. Pembrolizumab in advanced soft-tissue sarcoma and bone sarcoma (SARC028): a multicentre, two-cohort, single-arm, open-label, phase 2 trial. Lancet Oncol 2017;18:1493-501.

13 Jain RK. Antiangiogenesis strategies revisited: from starving tumors to alleviating hypoxia. Cancer Cell 2014;26:605-22.

14 Lin C, Wang S, Xie W, et al. Apatinib inhibits cellular invasion and migration by fusion kinase KIF5B-RET via suppressing RET/Src signaling pathway. Oncotarget 2016;7:59236-44.

15 Zheng B, Ren T, Huang Y, et al. PD-1 axis expression in musculoskeletal tumors and antitumor effect of nivolumab in osteosarcoma model of humanized mouse. J Hematol Oncol 2018;11:16.

16 Ramjiawan RR, Griffioen AW, Duda DG. Anti-Angiogenesis for cancer revisited: is there a role for combinations with immunotherapy? Angiogenesis 2017;20:185-204

17 Tartour E, Pere H, Maillere B, et al. Angiogenesis and immunity: a bidirectional link potentially relevant for the monitoring of antiangiogenic therapy and the development of novel therapeutic combination with immunotherapy. Cancer Metastasis Rev 2011;30:83-95

18 Fang W, Yang Y, Ma Y, et al. Camrelizumab (SHR-1210) alone or in combination with gemcitabine plus cisplatin for nasopharyngeal carcinoma: results from two single-arm, phase 1 trials. Lancet Oncol 2018;19:1338-50.

19 Eisenhauer EA, Therasse P, Bogaerts J, et al. New response evaluation criteria in solid tumours: revised RECIST guideline (version 1.1). Eur J Cancer 2009;45:228-47.

20 de Kock I, Mirhosseini M, Lau F, et al. Conversion of Karnofsky performance status (KPS) and eastern cooperative Oncology Group performance status (ECoG) to palliative performance scale (PPS), and the interchangeability of PPS and KPS in prognostic tools. $J$ Palliat Care 2013;29:163-9.

21 Steliga M, Vaporciyan A. Surgical treatment of pulmonary metastases from osteosarcoma in pediatric and adolescent patients. Cancer Treat Res 2009;152:185-201.

22 Seymour L, Bogaerts J, Perrone A, et al. iRECIST: guidelines for response criteria for use in trials testing immunotherapeutics. Lancet Oncol 2017;18:e143-52.

23 Cocks K, King MT, Velikova G, et al. Evidence-Based guidelines for determination of sample size and interpretation of the European organisation for the research and treatment of cancer quality of life questionnaire core 30. J Clin Oncol 2011;29:89-96.

24 Nicolini A, Carpi A, Rossi G. Relationship of cellular immunity, cytokines and CRP with clinical course in breast cancer patients with endocrine-dependent distant metastases treated with immunotherapy. Cancer Lett 2007;251:330-8.

25 Jeyakumar G, Kim S, Bumma N, et al. Neutrophil lymphocyte ratio and duration of prior anti-angiogenic therapy as biomarkers in metastatic RCC receiving immune checkpoint inhibitor therapy. $J$ Immunother Cancer 2017;5:82.

26 Montazeri A, Harirchi I, Vahdani M, et al. The European organization for research and treatment of cancer quality of life questionnaire (EORTC QLQ-C30): translation and validation study of the Iranian version. Support Care Cancer 1999;7:400-6.

27 Bielack SS, Smeland S, Whelan JS, et al. Methotrexate, doxorubicin, and cisplatin (MAP) plus maintenance pegylated interferon alfa$2 b$ versus MAP alone in patients with resectable high-grade osteosarcoma and good histologic response to preoperative map: first results of the EURAMOS-1 good response randomized controlled trial. J Clin Oncol 2015;33:2279-87.

28 Navid F, Sondel PM, Barfield R, et al. Phase I trial of a novel anti-GD2 monoclonal antibody, Hu14.18K322A, designed to decrease toxicity in children with refractory or recurrent neuroblastoma. J Clin Oncol 2014;32:1445-52.

29 Collins M, Wilhelm M, Conyers R, et al. Benefits and adverse events in younger versus older patients receiving neoadjuvant chemotherapy for osteosarcoma: findings from a meta-analysis. $J$ Clin Oncol 2013;31:2303-12. 
Correction: Apatinib plus camrelizumab (anti-PD1 therapy, SHR-1210) for advanced osteosarcoma (APFAO) progressing after chemotherapy: a single-arm, open-label, phase 2 trial

Xie L, Xu J, Sun X, et al. Apatinib plus camrelizumab (anti-PD1 therapy, SHR-1210) for advanced osteosarcoma (APFAO) progressing after chemotherapy: a single-arm, openlabel, phase 2 trial. J Immunother Cancer 2020;8:e000798. doi: 10.1136/jitc-2020-000798.

Since the online publication of this article, the authors have noticed that in figure 4 the dotted and full lines were mislabelled. Please see the corrected Figure below:

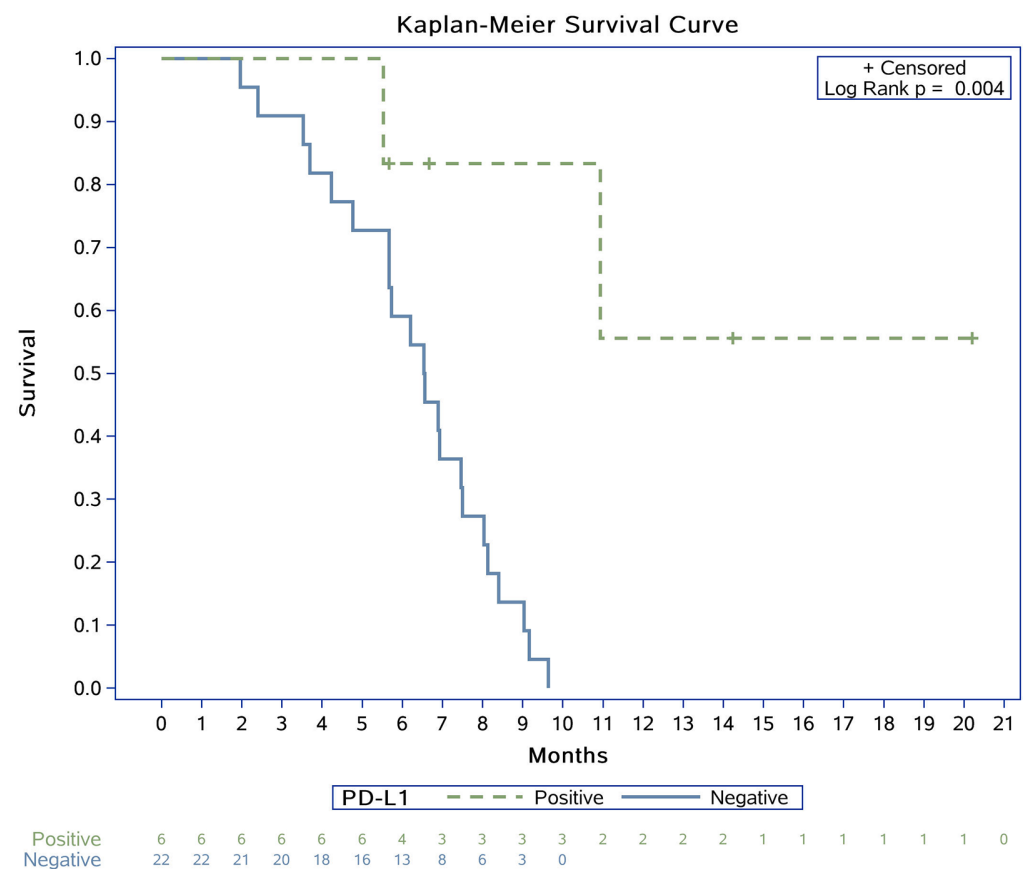

Figure 4

Open access This is an open access article distributed in accordance with the Creative Commons Attribution Non Commercial (CC BY-NC 4.0) license, which permits others to distribute, remix, adapt, build upon this work non-commercially, and license their derivative works on different terms, provided the original work is properly cited, appropriate credit is given, any changes made indicated, and the use is non-commercial. See http://creativecommons.org/licenses/by-nc/4.0/.

(c) Author(s) (or their employer(s)) 2020. Re-use permitted under CC BY-NC. No commercial re-use. See rights and permissions. Published by BMJ.

J Immunother Cancer 2020;8:e000798corr1. doi:10.1136/jitc-2020-000798corr1

D) Check for updates 